\title{
NOISE ANALYSIS OF MODULATED QUANTIZER BASED ON OVERSAMPLED SIGNALS
}

\section{Charlotte Yuk-Fan Ho}

Dept. of Electronic

Engineering and School of

Mathematical Sciences,

Queen Mary, University of

London, Mile End Road,

London, E1 4NS, U.K.

\section{ABSTRACT}

In this paper, a noise analysis of a modulated quantizer is performed. If input signals are oversampled, then the quantization error could be reduced by modulating both the input and the output of the quantizer. The working principle is based on the fact that convolutions of bandpass signals would spread wider in the frequency spectrum than that of lowpass signals. Hence, by filtering the high frequency components, the signal-to-noise ratio (SNR) could be increased. Numerical simulation results show that the modulated quantization scheme could achieve an average of $13.0960 \mathrm{~dB}$ to $21.4700 \mathrm{~dB}$ improvements on SNR over the conventional scheme, depends on the types of bandlimited input signals.

\section{INTRODUCTION}

Quantization is widely employed in many signal processing applications, such as in data compression [1] and analogto-digital conversion [2], etc. However, as quantization is not a reversible process because it is a many-to-one mapping, the system cannot be recovered once it is generated [3]. Hence, it is very important to minimize the quantization error.

The most common method to minimize the quantization error is based on the statistics of input signals [4]. Finer resolutions are assigned to the ranges of input signals which occur most frequently, and vice versa. However, this kind of quantization scheme requires a prior knowledge of statistics of inputs signals. In many situations, the statistics of input signals are unknown and this method cannot be applied directly.

Another common method to minimize the quantization error is via a sigma delta modulation technique [5]-[7]. If input signals are oversampled, then the signal band is very narrow. Hence, the overlap between the noise spectrum and the signal band is small. As a result, a very high SNR can be achieved. In this paper, we further utilize the oversampling technique to reduce the quantization error. The input and the output of a quantizer are modulated via a bank of modulators. Based on the obtained numerical simulation results, an average of $13.0960 \mathrm{~dB}$ to $21.4700 \mathrm{~dB}$ improvements on SNR over the conventional scheme can be achieved.

The outline of this paper is as follow: In Section 2, an approximated model for the quantizer is introduced. Based on the model, detail error analysis is performed. It is shown that the quantization error could be reduced by applying a modulation technique on the input and output of the quantizer. In Section 3, we further extend the results in Section 2 from a single modulator to a bank of modulators. Finally, a conclusion and future work is summarized and discussed in Section 4.

\section{REDUCTION OF NOISE VIA MODULATED QUANTIZER}

The block diagrams of systems using a conventional quantizer and a modulated quantizer are shown in, respectively, Figure 1a and Figure 1b. Denote the input to these two quantizers, the quantizers, the frequency response of these two linear time-invariant filters, the output of the conventional quantizer, that of the modulated quantizer, the reconstructed signal using the conventional quantizer, and that of using the modulated quantizer as, respectively, $u(k)$, $Q(\cdot), H(\omega), s_{1}(k), s_{2}(k), y_{1}(k)$ and $y_{2}(k)$. We assume that $u(k)$ is oversampled. That means, most of the energy of $u(k)$ is within the frequency band $\left(-\frac{\pi}{R}, \frac{\pi}{R}\right)$, where $R$ is the oversampling ratio. Consider an $N$ bit quantizer with the quantization range $[-L, L]$. Then 


$$
Q(y) \equiv\left\{\begin{array}{cc}
\Delta \operatorname{sign}(y)\left(\operatorname{ceil}\left(\frac{|y|}{\Delta}\right)-\frac{1}{2}\right) & |y| \leq L, \\
\operatorname{sign}(y)\left(2^{N-1}-\frac{1}{2}\right) & |y|>L
\end{array}\right.
$$

where $\operatorname{sign}(y) \equiv \begin{cases}\frac{y}{|y|} & y \neq 0, \operatorname{ceil}(y) \text { denotes the rounding } \\ 0 & y=0\end{cases}$ operator towards the plus infinity, $\mid \cdot$ denotes the absolute operator, and $\Delta \equiv \frac{L}{2^{N-1}}$ is the step size of the quantizer. To approximate $Q(y)$ as a polynomial of $y$, denoting $\mathbf{y} \equiv\left[\begin{array}{llll}y & y^{3} & \cdots & y^{2 M-1}\end{array}\right]^{T}$ and $\mathbf{p} \equiv\left[\begin{array}{lll}p_{1} & \cdots & p_{M}\end{array}\right]^{T}$, where the superscript ${ }^{T}$ denotes the transpose operator, $p_{m}$ for $m=1,2, \cdots, M$ and $2 M-1$ are, respectively, the coefficients and the order of the polynomial of $y$, then $\mathbf{p}$ can be found via solving the optimization problem with the objective being minimizing the total absolute square difference between the actual quantizer and the approximated quantizer, that is:

$$
\min _{\mathbf{p}} \int_{-L}^{L}\left|\mathbf{y}^{T} \mathbf{p}-Q(y)\right|^{2} d y .
$$

The solution of this optimization problem is $\mathbf{p}=-\mathbf{A}^{-1} \mathbf{b}$, where $\mathbf{A} \equiv 2 \int_{-L}^{L} \mathbf{y} \mathbf{y}^{T} d y$ and $\mathbf{b} \equiv-2 \int_{-L}^{L} Q(y) \mathbf{y} d y$. Figure 2 shows examples of input-output relationships of actual quantizers with $L=1$ and the approximated quantizers $\mathbf{y}^{T} \mathbf{p}$ with $M=10$ for 1-bit, 2-bit and 8-bit cases. Figure 3 show the corresponding differences, that is $Q(y)-\mathbf{y}^{T} \mathbf{p}$. It can be seen from Figure 3 that the differences between the actual quantizers and the approximated quantizers get smaller and smaller as $N$ increases. Hence, the approximation is valid. Now, let's analyze the quantization noise using the above approximated model. That is, replacing the actual quantizer $Q(y)$ by the approximated quantizer $\mathbf{y}^{T} \mathbf{p}$. Denote the Fourier transform of $u(k), s_{1}(k), s_{2}(k), y_{1}(k)$ and $y_{2}(k)$ as, respectively, $U(\omega), S_{1}(\omega), S_{2}(\omega), Y_{1}(\omega)$ and $Y_{2}(\omega)$. Denote $U_{2 m-1}(\omega) \equiv U(\omega) * \cdots * U(\omega)$, where $*$ denotes the convolution operator and there are $2 m-1$ terms in $U_{2 m-1}(\omega)$. For the system with the conventional quantizer shown in Figure 1a,

$$
Y_{1}(\omega)=H(\omega) S_{1}(\omega) \approx H(\omega) \sum_{m=1}^{M} p_{m} U_{2 m-1}(\omega) .
$$

Since we assume that $u(k)$ is oversampled, $U(\omega)$ is approximately bandlimited within $\left(-\frac{\pi}{R}, \frac{\pi}{R}\right)$. As a result, $U_{2 m-1}(\omega)$ is approximately bandlimited within $\left(-\frac{(2 m-1) \pi}{R}, \frac{(2 m-1) \pi}{R}\right)$. However, since all these $M$ terms have zero center frequency, all the higher order terms are overlapped to the signal band $\left(-\frac{\pi}{R}, \frac{\pi}{R}\right)$. If we regard all higher order terms $(m \geq 2)$ as the quantization noise, then the quantization noise would corrupt the signal seriously. Since

$$
S N R \approx 10 \log _{10} \frac{\int_{-\frac{\pi}{R}}^{\frac{\pi}{R}}\left|H(\omega) p_{1} U(\omega)\right|^{2} d \omega}{\int_{-\frac{\pi}{R}}^{\frac{\pi}{R}}\left|H(\omega) \sum_{m=2}^{M} p_{m} U_{2 m-1}(\omega)\right|^{2} d \omega},
$$

if we further assume that $H(\omega)$ is an ideal lowpass filter

$$
\begin{aligned}
\text { with } H(\omega)= & \left\{\begin{array}{cc}
1 & |\omega|<\frac{\pi}{R} \\
0 & \text { otherwise }
\end{array}\right. \text {, then } \\
& S N R \approx 10 \log _{10} \frac{p_{1}^{2} \int_{-\frac{\pi}{R}}^{\frac{\pi}{R}}|U(\omega)|^{2} d \omega}{\int_{-\frac{\pi}{R}}^{\frac{\pi}{R}}\left|\sum_{m=2}^{M} p_{m} U_{2 m-1}(\omega)\right|^{2} d \omega},
\end{aligned}
$$

which would be quite low for the conventional quantizer. Now consider the system with modulators as shown in Figure 1b. Denote the input to the quantizer as $\tilde{u}(k)$ and $\tilde{U}_{2 m-1}(\omega) \equiv \tilde{U}(\omega) * \cdots * \tilde{U}(\omega)$, in which there are $2 m-1$ terms in $\tilde{U}_{2 m-1}(\omega)$. Then

$\tilde{U}_{2 m-1}(\omega)=\frac{U_{2 m-1}(\omega)}{2^{2 m-1}} * \sum_{r=0}^{2 m-1} \frac{(2 m-1) !}{r !(2 m-1-r) !} \delta\left(\omega+(2 m-2 r-1) \omega_{0}\right),(5 \mathrm{a})$

$S_{2}(\omega) \approx \sum_{m=1}^{M}\left(\frac{p_{m} U_{2 m-1}(\omega)}{2^{2 m-1}} * \sum_{r=0}^{2 m-1} \frac{(2 m-1) !}{r !(2 m-1-r) !} \delta\left(\omega+(2 m-2 r-1) \omega_{0}\right)\right),(5 b)$

and

$Y_{2}(\omega) \approx H(\omega) \sum_{m=1}^{M}\left(\frac{p_{m} U_{2 m-1}(\omega)}{2^{2 m}} * \sum_{r=0}^{2 m} \frac{(2 m) !}{r !(2 m-r) !} \delta\left(\omega+2(m-r) \omega_{0}\right)\right),(5 \mathrm{c})$

where ! denotes the factorial operator. Hence,

$S N R \approx 10 \log _{10} \frac{\int_{-\frac{\pi}{R}}^{\frac{\pi}{R}}\left|H(\omega) \frac{p_{1} U(\omega)}{2^{2}} * \sum_{r=0}^{2} \frac{(2) !}{r !(2-r) !} \delta\left(\omega+2(1-r) \omega_{0}\right)\right|^{2} d \omega}{\int_{-\frac{\pi}{R}}^{\frac{\pi}{R}}\left|H(\omega) \sum_{m=2}^{M}\left(\frac{p_{m} U_{2 m-1}(\omega)}{2^{2 m}} * \sum_{r=0}^{2 m} \frac{(2 m) !}{r !(2 m-r) !} \delta\left(\omega+2(m-r) \omega_{0}\right)\right)\right|^{2} d \omega}$

If $\omega_{0}$ are selected in such a way that $\omega_{0} \geq \frac{(2 M-1) \pi}{R}$, then the mirror signals $U_{2 m-1}\left(\omega+2(m-r) \omega_{0}\right)$ for $r=0,1, \cdots 2 m$ and for $m=1,2, \cdots M$ do not overlap each others in the frequency spectrum. Hence, (6a) can be further simplified as: 


$$
S N R \approx 10 \log _{10} \frac{\frac{p_{1}^{2}}{4} \int_{-\frac{\pi}{R}}^{\frac{\pi}{R}}|U(\omega)|^{2} d \omega}{\int_{-\frac{\pi}{R}}^{\frac{\pi}{R}}\left|\sum_{m=2}^{M} \frac{p_{m} U_{2 m-1}(\omega)(2 m) !}{2^{2 m}(m !)^{2}}\right|^{2} d \omega} .
$$

Since $2^{2 m}=\sum_{r=0}^{2 m} \frac{(2 m) !}{r !(2 m-r) !}$, if $\sum_{\substack{r=0 \\ r \neq m}}^{2 m} \frac{(2 m) !}{r !(2 m-r) !}>\frac{3(2 m) !}{(m !)^{2}}$, then $\frac{4(2 m) !}{2^{2 m}(m !)^{2}}<1$ and

$$
\int_{-\frac{\pi}{R}}^{\frac{\pi}{R}}\left|\sum_{m=2}^{M} p_{m} U_{2 m-1}(\omega)\right|^{2} d \omega>4 \int_{-\frac{\pi}{R}}^{\frac{\pi}{R}}\left|\sum_{m=2}^{M} \frac{p_{m} U_{2 m-1}(\omega)(2 m) !}{2^{2 m}(m !)^{2}}\right|^{2} d \omega
$$

By comparing (4b) to (6b), the modulated system will provide improvement on SNR compared to the conventional system.

To verify the approximations and the above analysis, we have performed some simulation results. Denote $u^{\prime}(k)$ by a random signal with zero mean uniform distribution between -1 and 1 . The bandlimited input is generated via filtering $u^{\prime}(k)$ through $H(\omega)$ and normalizing the maximum absolute value to 1 , that is $U(\omega)=\frac{U^{\prime}(\omega) H(\omega)}{K}$, where $U^{\prime}(\omega)$ is the Fourier transform of $u^{\prime}(k)$ and $K$ is selected such that $\max _{\forall k \geq 0}|u(k)|=1$. In the following simulation results, we choose an elliptic filter with the following transfer function:

$H(z)=\frac{10^{-4}\left(0.0761-0.2027 z^{-1}+0.1283 z^{-2}+0.1283 z^{-3}-0.2027 z^{-4}+0.0761 z^{-5}\right)}{1-4.8675 z^{-1}+9.4816 z^{-2}-9.2392 z^{-3}+4.5036 z^{-4}-0.8785 z^{-5}}$ as the ideal lowpass filter because this filter can be obtained easily from the Matlab toolbox. Also, the saturation level of the quantizer is selected as 1 , that is $L=1$. This is because of the normalization reason. Moreover, we select the oversampling ratio as $R=64$ because this is the most common value employed in industry. Figure 4 shows the improvements of SNR of the modulated quantizer over the conventional quantizer, where $S N R \equiv 10 \log _{10} \frac{\sum_{\forall k \geq 0}|u(k)|^{2}}{\sum_{\forall k \geq 0} \mid u(k)-y_{i}(k)^{2}}$ for $i=1,2$. It is worth noting that the equation for calculating SNR here is different from that in the previous Section because the one in the previous Section is based on the approximated model, while the one in this Section is from the definition. According to the simulation results, it can be seen from Figure $4 a$ that there is an average of $5.3136 \mathrm{~dB}$ improvement when $\omega_{0}=\frac{\pi}{R}$ and $5.6084 \mathrm{~dB}$ improvement when $\omega_{0} \geq \frac{3 \pi}{R}$, but there is no significant change on the improvement when the modulating frequency is higher than $\frac{3 \pi}{R}$. Compared to the theory we have developed, that is, if $\omega_{0} \geq \frac{(2 M-1) \pi}{R}$ and $\sum_{\substack{r=0 \\ r \neq m}}^{2 m} \frac{(2 m) !}{r !(2 m-r) !}>\frac{3(2 m) !}{(m !)^{2}}$, then the SNR could be improved, it is interesting to see from Figure 4a that when $\omega_{0} \geq \frac{3 \pi}{R}$ it is already enough to satisfy the condition. Besides, there is an average of $6.9007 \mathrm{~dB}$ improvement when $N=1$ and the average improvement drops monotonically and converges to $5.3070 \mathrm{~dB}$ when $N=16$. This is because as $N$ increases, the effects of nonlinearity decrease. As a result, the improvement based on the modulation technique will be less significant. Figure $4 \mathrm{~b}$ shows the corresponding results for a sinusoidal input $u(k)=\sin \left(\frac{2 \pi k}{3 R}\right)$ for $k \geq 0$. We choose this sinusoidal input because this operating frequency is the most common test frequency employed for the analogdigital conversion and the magnitude of the sinusoidal input is chosen to be 1 because of the normalization reason. It can be seen from Figure $4 \mathrm{~b}$ that there is an average of $5.3821 \mathrm{~dB}$ improvement when $\omega_{0}=\frac{\pi}{R}$ and $5.7670 \mathrm{~dB}$ improvement when $\omega_{0} \geq \frac{3 \pi}{R}$, but there is no significant change on the improvement when the modulating frequency is higher than $\frac{3 \pi}{R}$. This phenomenon occurs similarly for the bandlimited random input case. However, we observe that there is an average of $4.8981 \mathrm{~dB}$ improvement when $N=1$ and the average improvement increases and converges to $5.7619 \mathrm{~dB}$ when $N=16$ for the sinusoidal input.

\section{EXTENSION FROM A SINGLE MODULATOR TO A BANK OF MODULATORS}

The technique discussed in Section 2 can actually be further extended to the case if a bank of modulators is employed. Denote $N_{q}$ as the number of modulators employed in the system as shown in Figure 5. Figure 6 show simulation results of various quantizers with same values of $L, R$, and the filter as in the previous Section. It can be seen from Figure 6a that there is an average of $6.2730 \mathrm{~dB}$ improvement when $N_{q}=1$ and the average improvement increases and converges to $11.0943 \mathrm{~dB}$ when $N_{q}=40$. Besides, there is an average of $19.6950 \mathrm{~dB}$ improvement when $N=1$ and the average improvement decreases monotonically and converges to $9.4297 \mathrm{~dB}$ when $N=16$ for a bandlimited random input. Figure $6 b$ shows the 
corresponding results for a sinusoidal input. It can be seen from Figure $6 \mathrm{~b}$ that there is an average of $5.7679 \mathrm{~dB}$ improvement when $N_{q}=1$ and the average improvement increases and converges to $11.0636 \mathrm{~dB}$ when $N_{q}=40$. Besides, there is an average of $12.1371 \mathrm{~dB}$ improvement when $N=1$ and the average improvement decreases monotonically and converges to $10.3246 \mathrm{~dB}$ when $N=16$. According to the simulation results, it is found that the highest improvement occurs at $N=1$ and $N_{q}=30$ for both a bandlimited random input and a sinusoidal input. The corresponding improvements are $21.4700 \mathrm{~dB}$ and $13.0960 \mathrm{~dB}$, respectively.

\section{CONCLUSION}

In this paper, we propose to employ a bank of modulators for reducing the quantization error. Since bandpass signals spread wider in the frequency spectrum than that of the lowpass signals, quantization error could be reduced by filtering the high frequency components. Numerical simulation results show that an average of $13.0960 \mathrm{~dB}$ to $21.4700 \mathrm{~dB}$ improvements on SNR over the conventional scheme could be achieved. It is worth noting that this technique is different from the dithering approach because a signal is added to the quantizer output for the dithering approach, while we propose to multiply a signal at the input and the output of the quantizer.

\section{ACKNOWLEDGEMENTS}

The work obtained in this paper was supported by a research grant from Queen Mary, University of London.

\section{REFERENCES}

[1] E.H. Yang, and Z. Zhang, "An On-line Universal Lossy Data Compression Algorithm via Continuous Codebook Refinement—Part III: Redundancy Analysis," IEEE Transactions on Information Theory, vol. 44, no. 5, pp. 1782-1801, 1998.

[2] D.E. Quevedo, and G.C. Goodwin, "Multistep Optimal Analogto-Digital Conversion," IEEE Transactions Circuits and Systems - I: Regular Papers, vol. 52, no. 3, pp. 503-515, 2005.

[3] N.T. Thao, "Vector Quantization Analysis of $\Sigma \Delta$ Modulation," IEEE Transactions on Signal Processing, vol. 44, no. 4, pp. 808817, 1996.

[4] M.G. Strintzis, and D. Tzovaras, "Optimal Pyramidal Decomposition for Progressive Multidimensional Signal Coding Using Optimal Quantizers," IEEE Transactions on Signal Processing, vol. 46, no. 4, pp. 1054-1068, 1998.

[5] C.Y.F. Ho, B.W.K. Ling, J.D. Reiss, and X. Yu, "Nonlinear Behaviors of Bandpass Sigma Delta Modulators with Stable System Matrices," to appear in IEEE Transactions on Circuits and Systems-II: Express Briefs.

[6] C.Y.F. Ho, B.W.K. Ling, and J.D. Reiss, "Fuzzy Impulsive Control of High Order Interpolative Lowpass Sigma Delta Modulators," to appear in IEEE Transactions on Circuits and Systems-I: Regular Papers.
[7] C.Y.F. Ho, B.W.K. Ling, J.D. Reiss, Y.Q. Liu, and K.L. Teo, "Design of Interpolative Sigma Delta Modulators via Semi-infinite Programming," to appear in IEEE Transactions on Signal Processing.

$$
\stackrel{u(k)}{\longrightarrow} Q \stackrel{s_{1}(k)}{\longrightarrow} H(\omega) \stackrel{y_{1}(k)}{\longrightarrow}
$$

Figure 1a. A system using conventional quantizer.

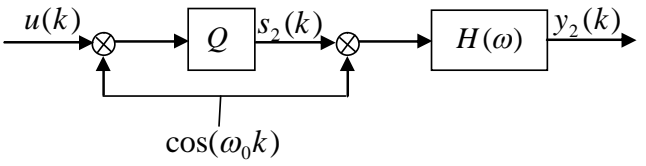

Figure $1 \mathrm{~b}$. A system using modulated quantizer.

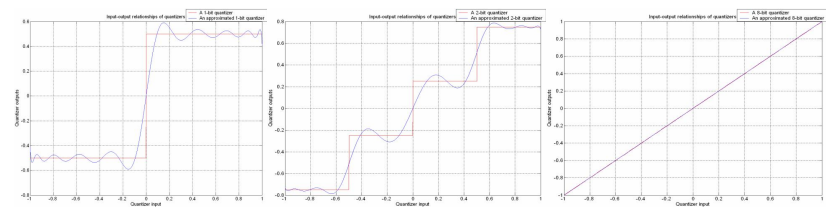

Figure 2. Input-output relationships of the original quantizers and the approximated quantizers. (a) 1-bit case. (b) 2-bit case. (c) 8-bit case.

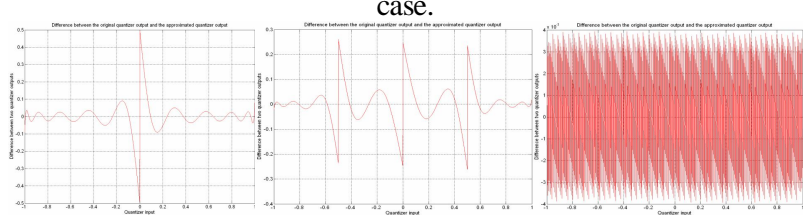

Figure 3. Differences between the original quantizers and the approximated quantizers. (a) 1-bit case. (b) 2-bit case. (c) 8-bit case.
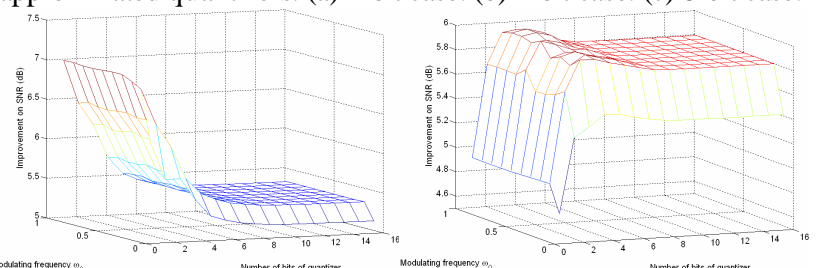

Figure 4. Effect of different number of bits of quantizers and modulating frequencies on the improvements of SNR. (a) a bandlimited random input. (b) a sinusoidal input.

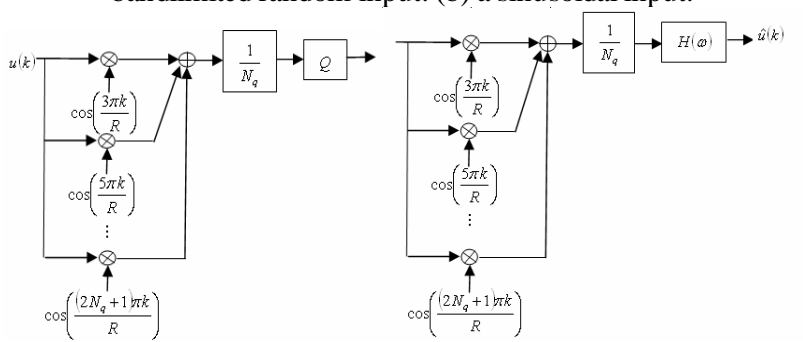

Figure 5. A system for noise reduction using a bank of modulators.
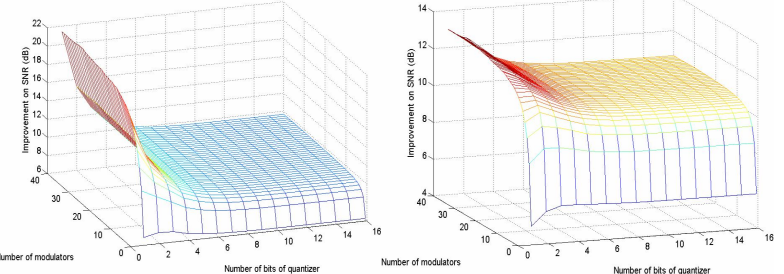

Figure 6. Effect of different number of bits of quantizers and number of modulators on the improvements of SNR. (a) a bandlimited random input.(b) a sinusoidal input. 